\title{
Valores normales de olfato, hiposmia y anosmia en población chilena sana según la batería "sniffin sticks"
}

Unidad Trastornos del Movimiento, Departamento de Neurología-Neurocirugía Hospital Clínico Universidad de Chile. Santiago de Chile.

Sin fuentes de financiamiento.

Recibido el 20 de junio de 2011, aceptado el 12 de diciembre de 2011

Correspondencia a:

Dr Pablo Venegas Francke Av. Apoquindo 4100, oficina 609

Fono: 2123157

E-mail: Pablo.venegasf@ gmail.com

\author{
LORENA HUDSON, M. CONSUELO SILVA, JUAN CRISTÓBAL NÚÑEZ, \\ RODRIGO GÓMEZ, PABLO VENEGAS-FRANCKE
}

\section{Evaluation of olfaction in healthy subjects using the sniffing sticks battery}

Background: Olfaction dysfunction is linked to neurodegenerative diseases, therefore the evaluation of this function is becoming important Aim: To evaluate olfaction in healthy participants. Material and Methods: We evaluated 44 healthy males and 55 females, aged 21 to 89 years with the sniffing sticks battery to determine normal values in Chilean population. During the test, participants must correctly identify 12 different odors. Results: Normal olfaction, hyposmia and anosmia were defined. An age related decline in olfaction was observed, especially among males aged 59 years or more. Conclusions: This study provides age and gender specific normal values for the sniffing sticks battery.

(Rev Med Chile 2012; 140: 442-446).

Key words: Aged; Gender; Smell.
L a evaluación del sentido del olfato ha cobrado una gran importancia en las últimas décadas dada su relación con enfermedades neurodegenerativas y de las condiciones fisiológicas asociadas a su alteración ${ }^{1}$.

Se han caracterizado tres componentes de la olfacción que se alteran en distinta intensidad según la causa subyacente: la percepción del olor a bajas concentraciones (umbral), la habilidad para nombrar o asociar un olor (identificación) y la distinción no verbal de distintos olores (discriminación). Esta última requiere el uso de la memoria operativa, ya que los estímulos olfatorios, al contrario de lo que sucede en la modalidad visual o auditiva, no pueden compararse simultáneamente, sino en orden secuencial ${ }^{2}$.

Un estudio demostró que la prevalencia de la disfunción olfativa alcanza $1,4 \%$, en la población general, pero se cree que esta patología es subdiagnosticada ${ }^{3}$. Múltiples estudios han demostrado que existe un déficit olfativo progresivo asociado a la edad, el cual se debe a cambios neuropatológicos propios del envejecimiento ${ }^{4-6}$. Cain y Stevens mostraron que, en el olfato, los umbrales sensoriales comienzan a debilitarse progresivamente a partir de la cuarta década de vida debido a que existe un incremento de los umbrales absolutos para una gran variedad de sustancias, una disminución en la capacidad de percibir la intensidad de un olor y una menor capacidad de identificar olores ${ }^{7}$. Diferentes investigaciones han determinado el grado de pérdida de la olfación en población general según edad, cifras que van desde 1,99\% entre los 55 a 64 años, 2,6\% entre 65 a 74 años y de 4,6\% sobre los 75 años $^{3}$. Dicha alteración ocurre también en numerosas enfermedades neurológicas, principalmente en las enfermedades neurodegenerativas como la enfermedad de Parkinson (EP) y la enfermedad de Alzheimer ${ }^{8}$.

La EP es, en cuanto a prevalencia, la segunda enfermedad neurodegenerativa luego de la enfermedad de Alzheimer y su asociación con una alteración del sentido del olfato fue reportada por primera vez hace alrededor de 30 años. 
La EP se manifiesta clínicamente con bradicinesia, rigidez muscular, inestabilidad postural y/o temblor de reposo, elementos necesarios para realizar el diagnóstico de EP según los Criterios del Banco de Cerebros de la Sociedad de EP del Reino Unido, sin embargo, estas manifestaciones son tardías. Existen numerosos síntomas y signos que pueden orientar más precozmente a un diagnóstico de EP como son las alteraciones del sueño en la fase de movimientos oculares rápidos (sueño REM por el acrónimo inglés), trastornos de ánimo y ansiedad, disautonomía y déficit olfatorio. Este último constituye uno de los primeros y más frecuentes síntomas premotores de la enfermedad. $\mathrm{Su}$ base patológica radica en la pérdida neuronal precoz de las vías olfatorias del bulbo olfatorio y núcleos olfatorios bulbares, aun antes del compromiso mesencefálico que explica la aparición de los síntomas motores.

Es por esto que ha surgido un creciente interés por la exploración del sentido del olfato y en los últimos años se han desarrollado varios tests olfatorios, entre los que se encuentran el "University of Pennsylvania Smell Identification Test” (UPSIT) ${ }^{9}$, el "San Diego Odor Identification Test"10, y el "Scandinavian Odor Identification Test"11. Estos tests emplean un rango de estímulos que varía entre 3 y 40 y son de respuesta múltiple. Otros tests que evalúan distintas funciones olfatorias son el "Connecticut Chemosensory Clinical Research Center Tests" 12 para la detección e identificación olfatoria, y el "Sniffin' Sticks Test", desarrollado por Hummel en Alemania, el cual mide intensidad, discriminación e identificación mediante la olfacción de veinte esencias. Una variación de éste último es el "Sniffin Sticks Screening Test", desarrollado por el mismo autor, el cual evalúa la identificación de doce aromas. Se considera normal para población europea obtener diez o más puntos, hiposmia entre nueve y seis y anosmia un puntaje menor o igual a cinco $^{13}$. Este último test es fácil aplicación y dado los aromas utilizados es aplicable a poblaciones de distintos acervos culturales, incluyendo población chilena.

Cabe destacar que dichas investigaciones se han llevado a cabo en población extranjera, utilizando los rangos normales de olfacción de dicha población. En nuestro medio se utiliza como uno de los estudios complementarios en el diagnóstico de la EP, sin embargo, no se dispone de valores de normalidad en sujetos sanos.
El objetivo de nuestro estudio es determinar los valores de olfacción normal en población chilena usando "Sniffin Sticks Screening Test" y así validar dicha prueba ajustada a nuestra población en el estudio diagnóstico de Enfermedad de Parkinson en etapas precoces de la enfermedad.

\section{Materiales y Métodos}

Se obtiene una muestra, previo consentimiento informado, de 99 voluntarios sanos seleccionados aleatoriamente y representativos de la población chilena en género y grupo etario de acuerdo a distribución del último censo nacional (2002).

Constituyen criterios de inclusión pacientes adultos, capaces de colaborar con el examen; y criterios de exclusión EP, depresión severa actual, deterioro cognitivo evidente evaluado clínicamente al momento del examen, infección rino-sinusal aguda o crónica, antecedente de TEC grave y tabaquismo crónico; dado que estos cuadros pueden interferir en la capacidad normal de olfacción. Dentro de la muestra hubo pacientes con comorbilidades médicas como hipertensión arterial y diabetes mellitus.

Se les aplica el "Sniffin Sticks Screening Test", consistente en la utilización de doce lápices aromáticos con esencias de olores disueltos en polipropilenoglicol: naranja, cuero, canela, menta, plátano, limón, anís, café, clavo de olor, piña, rosa, pescado. Estos fueron presentados de forma secuencial al sujeto durante tres segundos a dos centímetros de distancia de las fosas nasales y se le solicitó al paciente que identifique el aroma eligiendo entre cuatro alternativas posibles leídas por el examinador. Se presentan las sucesivas esencias con un intervalo de al menos 30 segundos entre cada uno. Se otorga un puntaje según el total de aromas reconocidos.

Se determina el valor normal del resultado de la técnica en sujetos sanos de la población chilena mediante análisis estadístico con prueba de Kolmogorov-Smirnov y Shapiro-Wilk.

\section{Resultados}

Se evaluaron 99 sujetos que corresponden a 55 mujeres (56\%) y 44 hombres (44\%), comprendidos entre los 21 y 89 años. Se clasifican en tres subgrupos según rango etario: grupo I de 21 a 39 
años (G1), grupo II de 40 a 59 años (G2) y grupo III mayor o igual a 60 años (G3) (Tabla 1).

Se evaluó cada esencia de forma independiente obteniendo el siguiente resultado expresado como porcentaje de respuestas correctas: naranja $80 \%$, cuero $84 \%$, canela $57 \%$, menta $95 \%$, plátano $98 \%$, limón $46 \%$, anís $89 \%$, café $86 \%$, clavo de olor $88 \%$, piña $88 \%$, rosa $90 \%$, pescado $99 \%$ (Figura 1).

El número de respuestas correctas presentó una distribución de Gauss, según prueba de normalidad de Kolmogorov-Smirnov (mediana: 10, desviación estándar: 1,49) (Figura 2). A partir de esta distribución se definieron los valores de normalidad/hiposmia/anosmia utilizando la desviación estándar. Del total de pacientes, 68 pacientes obtuvieron un puntaje entre 8 y 12 (69\%), 29 pacientes obtuvieron un puntaje entre 6 y $7(29 \%)$ y 2 pacientes obtuvieron un puntaje menor o igual a $5(2 \%)$.

Se realizó análisis de acuerdo a grupos etarios antes definidos. El grupo etario I (21 a 39 años) presentó una mediana de 11 y una desviación estándar de 1,16, el grupo etario II (40 a 59 años) presentó una mediana de 10 y una desviación estándar de 1,30 y el grupo etario III (sobre 59 años) presentó una mediana de 10 y una desviación estándar de 1,91. (Tabla 2). Considerando lo anterior se definieron rangos de normalidad según grupo

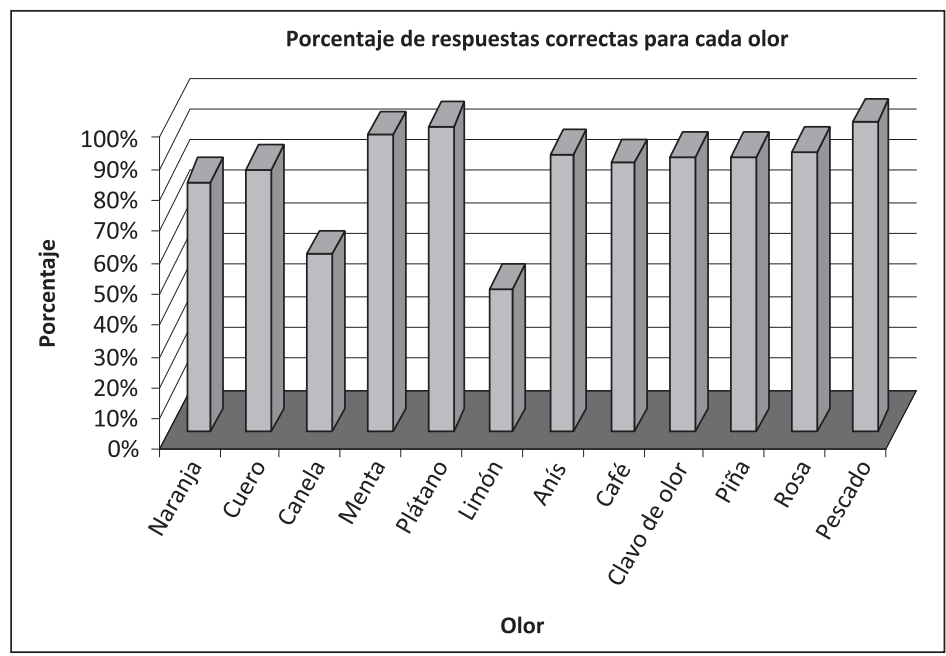

Figura 1. Gráfico de respuestas correctas en cada aroma. Nótese la caída en aromas de canela y limón, en el resto de los aromas el acierto es superior al $80 \%$.

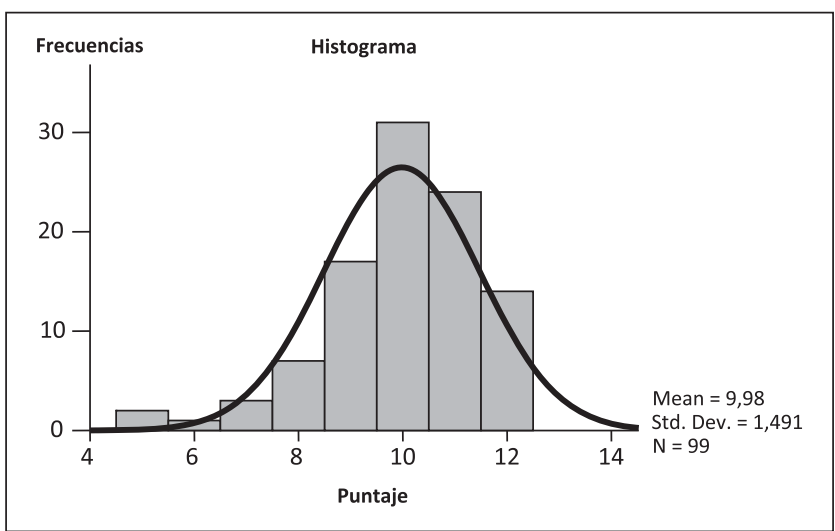

Figura 2. Gráfico de distribución de respuestas correctas. Nótese distribución normal, lo que permite definir patrones de normalidad de acuerdo a desviaciones estándar.
Tabla 1. Características de la muestra analizada. Anexo

\begin{tabular}{|lcc|}
\hline & Hombres & Mujeres \\
\hline 21 a 39 años & 21 & 25 \\
\hline 40 a 59 años & 16 & 19 \\
\hline$\geq 6$ años & 7 & 11 \\
\hline Total & 44 & 55 \\
\hline
\end{tabular}

La muestra es representativa en cuanto a distribución por género y edad según datos de último censo. 
Valores normales de olfacción en Chile - L. Hudson et al

Tabla 2. Resultados de olfacción, de acuerdo a grupo etario

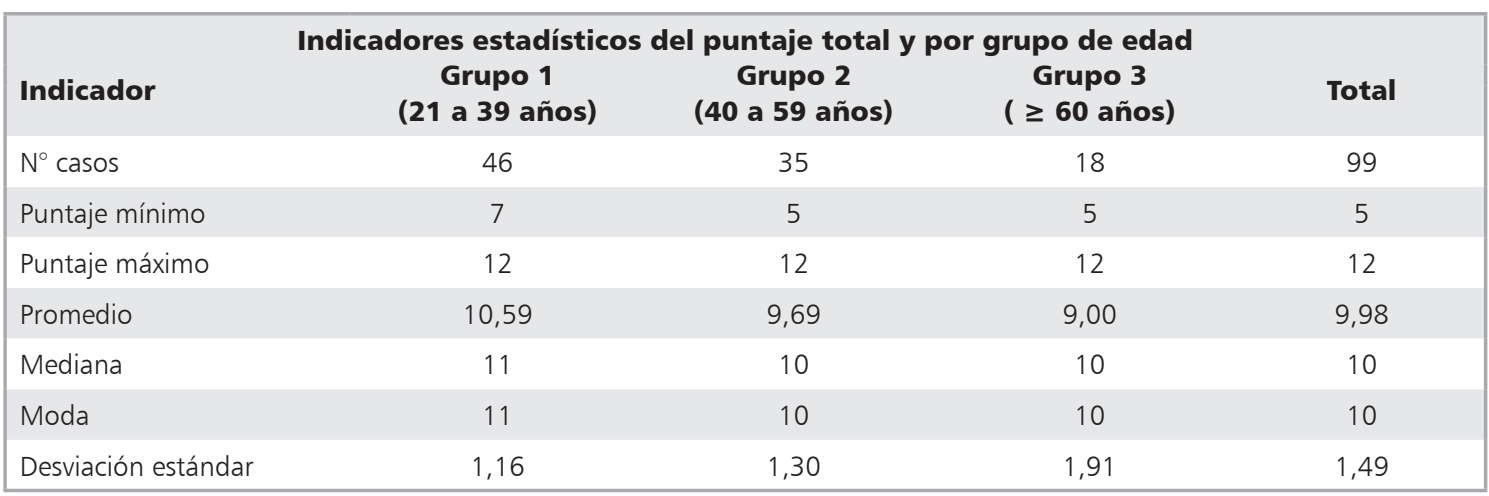

La tabla muestra los valores estadísticos de los resultados de acuerdo a cada grupo etario.

etario (Tabla 3). Se consideró hiposmia cuando el rendimiento estuvo entre -1 y -2 desviaciones estándares y anosmia cuando el rendimiento fue bajo 2 desviaciones estándares.

\section{Discusión}

El creciente interés respecto a los procesos fisiológicos y patológicos que afectan el sentido del olfato y su ampliamente demostrada correlación con enfermedades neurodegenerativas, han llevado a desarrollar diversas técnicas para objetivar su déficit de modo de utilizarlo en la detección precoz de estas enfermedades ${ }^{14}$.

En nuestro estudio se evalúa la discriminación de doce aromas en población chilena sana utilizando el "Sniffin Sticks Screening Test", el cual, hasta la fecha se basaba en valores de normalidad de sensibilidad olfativa en pacientes sanos de población europea.

La población analizada en nuestro estudio demuestra un menor rendimiento en el reconocimiento de los aromas de canela y limón. Este hallazgo cuya causa no podemos precisar, no se debería a un factor cultural, pues son aromas familiares en nuestro medio.

Estudios previos con el uso de esta y otras técnicas, han determinado que los niveles de olfacción van disminuyendo con la edad en ambos sexos y que los hombres en condiciones fisiológicas huelen menos que las mujeres. En nuestro estudio se corrobora el hecho de que en ambos géneros los valores de normalidad disminuyen con la edad.
Tabla 3. Valores de referencia de acuerdo a grupo etario

\begin{tabular}{|lccc|}
\hline \multicolumn{4}{c|}{ Resultados } \\
& Normal & Hiposmia & Anosmia \\
\hline Grupo 1 & 9 a 12 & 7 a 8 & $\leq 6$ \\
Grupo 2 & 8 a 12 & 6 a 7 & $\leq 5$ \\
Grupo 3 & 7 a 12 & 5 a 6 & $\leq 4$ \\
Total & 8 a 12 & 6 a 7 & $\leq 5$ \\
\hline
\end{tabular}

Se muestra los valores normales, hiposmia (menos una desviación estándar) y anosmia (menos dos desviaciones estándar) de la población total y según grupo etario.

Los hombres tienen valores de olfacción igual a las mujeres excepto en el grupo mayor o igual de 60 años, donde se observan valores de normalidad menores en hombres que en mujeres, pero debido a la escasa representación de dicho segmento respecto al total de la muestra, este hallazgo necesita ser replicado para reafirmar su validez. A la vez existe un aumento progresivo del total de sujetos con hiposmia (definida ésta según valores de normalidad de la población total) en los grupos etarios dos y tres, todo lo cual se debería a un envejecimiento fisiológico.

Este estudio otorga valores de normalidad de la población chilena general y de acuerdo a grupos etarios, para el test "sniffin sticks", lo que puede facilitar una mejor interpretación al momento de analizar los resultados de un paciente particular. 


\section{Referencias}

1. Landis B, Knecht M, Hüttenbrink K, Lacroix J, Humel T. Clinical aspects of dysosmia and presentation of European Olfactory Test of "sniffin sticks": a review. J Otolaryngol 2005; 34 (2): 86-92.

2. Fusari A, Molina J. Olfato, envejecimiento fisiológico y enfermedades neurodegenerativas: II. Envejecimiento y enfermedades neurodegenerativas. Rev Neurol 2009; 49: 363-9.

3. Hoffman HJ, Ishii EK, Mac Turk RH. Age-related changes in the prevalence of smell/taste problems among the United States adult population. Results of the 1994 disability supplement to the National Health Interview Survey (NHIS). Ann N Y Acad Sci 1998; 30: 716-22.

4. Stevens J, Dadarwala A. Variability of olfactory threshold and its role in assessment of aging. Percept Psychophys 1993; 54: 296-302.

5. Murphy C, Schubert C, Cruickshanks K, Klein B, Klein $\mathrm{R}$, Nondahl D. Prevalence of olfactory impairment in older adults. JAMA 2002; 288: 2307-12.

6. Schiffmann S. Olfaction in aging and medical disorders. En Serby M, Chobor K, editores. Science of olfaction. New York, USA: Springer; 1992. p. 500-25.

7. Cain W, Stevens J. Uniformity of olfactory loss in aging. Ann NY Acad Sci 1989; 561: 29-38.
8. Katzenschlager R, Lees A. Olfaction and Parkinson's disease: its role in differential diagnosis. Curr Opin Neurol 2003; 17: 417-23.

9. Doty R, Shaman P, Dann M. Development of the University of Pennsylvania Smell Identification Test: a standardized microencapsulated test of olfactory function. Physiol Behav 1984; 32: 489-502.

10. Anderson J, Maxwell L, Murphy C. Odorant identification testing in the young child. Chem Senses 1992; 17: 590.

11. Nordin S, Brämerson A, Lidén E, Bende M. The Scandinavian Odor Identification Test: development, reliability, validity and normative data. Acta Otolaryngol 1998; 118: 226-34.

12. Cain W, Goodspeed R, Gent JF, Leonard G. Evaluation of olfactory dysfunction in the Connecticut Chemosensory Clinical Research Center. Laryngoscope 1988; 98: 83-8.

13. Hummel T, Sekinger B, Wolf S, Pauli E, Kobal G. 'Sniffin sticks': olfactory performance assessed by the combined testing of odor identification, odor discrimination and olfactory threshold. Chem Senses 1997; 22 (1): 39-52.

14. Eibenstein A, Fioretti A, Lena C, Rosati N, Ottoviano I, Fusseti M. Olfactory screening test: experience in 102 Italian subjects. Acta Otorhinolaryngol Ital 2005; 25 (1): 18-22. 